\title{
Introduction to the Linear Theory of Tearing Instabilities
}

February 1978

Prepared for

U.S. Department of Energy

Assistant Secretary for Energy Technology Office of Fusion Energy

Under Contract No. EY-76-C-05-4478 


\section{DISCLAIMER}

This report was prepared as an account of work sponsored by an agency of the United States Government. Neither the United States Government nor any agency Thereof, nor any of their employees, makes any warranty, express or implied, or assumes any legal liability or responsibility for the accuracy, completeness, or usefulness of any information, apparatus, product, or process disclosed, or represents that its use would not infringe privately owned rights. Reference herein to any specific commercial product, process, or service by trade name, trademark, manufacturer, or otherwise does not necessarily constitute or imply its endorsement, recommendation, or favoring by the United States Government or any agency thereof. The views and opinions of authors expressed herein do not necessarily state or reflect those of the United States Government or any agency thereof. 


\section{DISCLAIMER}

Portions of this document may be illegible in electronic image products. Images are produced from the best available original document. 


\section{Introduction to the Linear Theory of Tearing Instabilities}

Februạry 1978

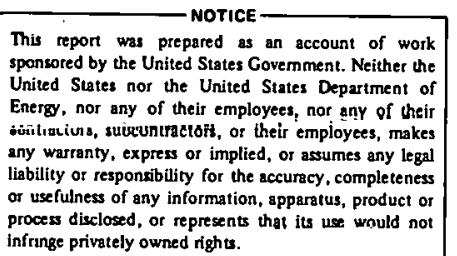

R.D. Hazeltine

Prepared for

U.S. Department of Energy

Assistant Secretary for Energy Technology Office of Fusion Energy

Under Contract No. EY-76-C-05-4478 


\section{NOTICE}

This report was prepared by The University of Texas as an account of work sponsored by the United States Government. Neither the United States nor the United States Department of Energy, nor any of theır employees, llu dily of their contractors, subcontractors, nor any of their employees, nor any of their contractors, subcontraclors, or their employees, makes any warranty, express or implied, or assumes any legal liability or responsibility for the accuracy, completeness, or usefulness of any information, apparatus, product or process disclosed, or represents that its use would not infringe privately owned rights.

\section{Avallable from:}

National Technical Information Service (NTIS) U.S. Department of Commerce

5285 Port Royal Road

Springfield, Virginia 22161

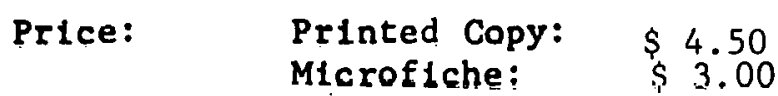


Introduction to the Linear Theory of Tearing Instabilities

R.D. Hazeltine

$\checkmark$ Fusion Research Center $\quad 950^{3 * 17}$

The University of Texas at Aus tin

Austin, Texas 78712 


\section{Introduction}

Revived interest calls for an introductory survey of tearing instabllity theory. Here, we try to make the relevant literature more widely and painlessly accessible, by summarizing the basic notions of the theory from an elementary point of view. We take advantage of the fact that most tearing theories, despite their often qualitatively different predicted growth rates and tearing layer widths, are very similar. Thus one can proceed surprisingly far in understanding tearing mode theory, without being committed to a specific model or ordering.

A complete review is not attempted. In particular, we restrict our attention lu lokamak-fusion applications, and recent nonlinear tearing studles are virtually ignored.

The reasons why tearing instabilities might bear importantly on tokamak performance are considered in Sec. II. Sec. III describes the mechanism of tearing, and Sec. IV outlines the method by which this mechanism is analyzed. We close in Sec. V with a survey of typlcal growth rate predictions.

Although the notion of magnetic field-line tearing apparently originated in the astrophysical literature, ${ }^{1}$ most fusion-oriented theories of tearing can be traced to the famous 1963 paper by Furth, Killeen and Rosenbluth (FKR). ${ }^{2}$ FKR treated a number of resistive instabilities, In the limit of vanishing gyration radius. But it was recognized ${ }^{3}$ that finite gyroradius effects, and other corrections to Ohm's law, would become significant as the plasma temperature and electrical conductivity increased, and such corrections were studied in several papers, by Coppi and collaborators, 4 and others, ${ }^{5,6}$ during the following two years. 
The subsequent five or six year lapse in tearing-related publlcations was perhaps a consequence of the apparently esoteric, "theoretical" nature of the modes, and their relatively slow growth rates (proportional to a fractional power of the resistivity). The revival of interest, beginning in 1971 and continuing to the present, was stimulated mainly by the tokamak experimental program: it was noticed ${ }^{7}$ that certain tokamak phenomena, univers ally observed, ${ }^{8}$ and seemingly assoclated with grave effects on confinement, had significant features in common with the predicted properties of tearing instabilities. Thus, in particular, the (now "classical") FKR stability criterion was studied in detall for varlous tokamak current profiles. ${ }^{9}$ The relation between tearing modes and the ideal MHD kink was also clarlfied ${ }^{10}$ (the experimental results having also stimulated new interest in ideal effects ${ }^{11,12}$ ). But most theoretical work during the 1970's has been concentrated within three areas: (1) Attempts to extend tearing mode theory to the long mean free path regimes of modern tokamak experiments. ${ }^{13,14,15}$ This effort has produced, Inter alla, substantial unification of previous work. ${ }^{14,15}$ (11) Investigations of the effects of toroldal curvature, both In the context of iesistive fluid theory, ${ }^{16}$ and also, most recently, in the long mean free path $11 \mathrm{~m} 1 \mathrm{t} .{ }^{17}$ Such effects turn out to be quite Important, as is also the case for Ideal kink modes. ${ }^{18}$,(iii) Studies, both analytical ${ }^{19}, 20$ and numerical, $21,22,23$ of the nonlinear properties of resistive instabilities. The objectives here Include theoretical understanding of the so-called disruptive Instabillty, 24,25 the consequences of magnetic island formation, ${ }^{26}$ and the effects of tearing instabilities on transport. 27,28 


\section{Significance of tearing instabllities}

A. Confinement

Plasma confinement in an axisymmetric, toroldal system ${ }^{29}$ depends upon the existence of nested surfaces of constant pressure $p$, almost each of which is covered ergodically by a single field line:

$$
\stackrel{\mathrm{B}}{\sim} \cdot \nabla \mathrm{p}=0 \text {. }
$$

Loss of confinement then requires some scattering mechanism to allow particle diffusion across the surfaces. But this radial motion is very slow: of order $(\nu / \Omega)^{2}$, where $\nu$ is the $90^{\circ}$-scattering frequency and $\Omega=e B / m c$ the gyrofrequency, compared to particle motion along the magnetic fleld.

Much more rapid loss of confinement can result from destruction of the "flux surfaces" themselves, due to radlal perturbations of the field. Consider a circular-cross-section tokamak, with toroldal coordinates $(r, \theta, \varphi): r$ is the minor radius, and $\theta(\varphi)$ is the pololdal (toroldal) angle. The unperturbed state is characterized by

$$
\partial p_{0} / \partial \varphi=0=B_{o r} \cdot
$$

Then Eq. (1) with $B_{\theta} \neq 0$ implies that $p_{0}$ depends only on $r$. For simpllcity, we consider perturbations $(\widetilde{\mathrm{p}}, \widetilde{\mathrm{B}})$ which maintain Eq. (1), while breakIng the symmetry of Eq. (2) (as would correspond to low frequency excitations, or "neighboring equilibria"). Periodicity allows us to expand

$$
\begin{aligned}
& -i \tilde{B}_{r}=\sum_{m, n} \psi_{m n} e^{i(m \theta-n \varphi)}, \\
& \tilde{p}=\sum \tilde{p}_{m n} e^{i(m \dot{\theta}-n \varphi)},
\end{aligned}
$$

and to express the linear version of Eq. (1) as 


$$
\tilde{p}_{m n}=-\left(r p_{o}^{\prime} / B_{O A}\right) \psi_{m n} /(m-n q) \text {, }
$$

where

$$
q(r) \equiv r B_{O \varphi} / R B_{O \theta}
$$

is the safety factor of the unperturbed system, and finite aspect ratio corrections are omitted. Evidently, the linear theory breaks down on flux surfaces satisfying

$$
\left|\left(r \mathrm{p}_{0}{ }^{\prime} / \mathrm{p}_{\mathrm{o}}\right)\left(\psi_{\mathrm{mn}} / \mathrm{B}_{\mathrm{o}}\right)\right| \geq|\mathrm{m}-\mathrm{nq}(\mathrm{r})| \text {. }
$$

For chosen integers $m$ and $n, E q$. (6) will describe a three-dimensional reglon,

$$
\left|r-r_{s}\right| \leq \Delta_{m n}
$$

where $r_{s}(m, n)$ labels the appropriate rational flux surface,

$$
q\left(r_{s}\right)=m / n
$$

and the radial width, $\Delta_{m n}$, can be seen to depend upon the shear, $q^{\prime} / q$, and upon the magnitude of the field perturbation at $r_{S}$. Inside this singular region, the perturbed constant pressure surfaces need not resemble their unperturbed counterparts. Furthermore, if Eq. (6) is satisfied for one pair $(m, n)$, it will in general be satisfied for numerous pairs $\left(m^{\prime}, n^{\prime}\right)$, with $\mathrm{m}^{\prime} / \mathrm{n}^{\prime} \approx \mathrm{m} / \mathrm{n}$, so that the pressure surfaces inside $\Delta_{\mathrm{mn}}$ can become chaotically scrambled: one has in this case a "flux-volume", in which the concept of flux surfaces is irrelevant, and confinement is locally absent.

On the other hand, for a fixed, reasonably smooth field perturbation $\left|B_{r}{ }^{\prime} B_{\theta}\right| \ll 1$, the width $\Delta_{m n}$ decreases sharply with increasing $m$ or $n$. [This follows from the presumed convergence of the series in Eq. (3).] Thus the presence of high-order rational values of $q$, and the fact that every 
number is nearly rational, are not physically important. One expects significant destruction of the irrational flux surface having $q=(1.1)^{\frac{1}{2}}$, but not, usually, of the rational surface having $q=23 / 16$.

Hence we may restrict our attention to reasonably small values of $\mathrm{m}$ and $\mathrm{n}$, so that on "most" flux surfaces, Eq. (6) is not satisfied. Such flux surfaces suffer only mild deformation, without topological change. The situation for both types of surface is deplcted in Fig. 1.

These remarks are made precise in a theorcm ("ISAM") Ilıt conjectured by Kolmogorov, and later proved by Arnol'd and Moser, in the context of ergodic theory. An illuminating discussion has been presented by Walker and Ford. ${ }^{30}$ The significance of the KAM theorem to plasma confinement was pointed out by Grad, ${ }^{31}$ while detailed studies of flux surface destruction have been presented by, among others, Rosenbluth et al. ${ }^{32}$ and, most recently, Rechester and Stix. ${ }^{26}$

Ideal MHD theory ${ }^{11}$ predicts that the most dangerous modes in a tokamak have toroldal mode number $\mathrm{n}=1$, so that flux surface destruction, and local loss of confinement, seems most likely to occur near flux surfaces having small integral safety factors: $q=1,2,3 \ldots$. But 1deal MHD also predicts that

$$
B_{r}\left(r_{S}\right)=0
$$

in which case Eq. (6) cannot be satisfled and the flux surface topology is preserved!

This comment brings us, finally, to the subject of tearing, since the crucial property of a tearing mode is the presence of a radial magnetic field perturbation on rational flux surfaces. Thus any observed flux surface destruction (if it results from an instability rather than lack of equilibrium ${ }^{31}$ ) 
can be associated with something akin to a tearing instabllity.

It follows that tearing instabilities could provide fundamental limitations on tokamak confinement: they could limit, for example, the maximum achievable $\beta \equiv 8 \pi p / B^{2}$. When the singular regions of Eq. (7) are well separated, the resulting local confinement loss would appear in the observed pressure or temperature profiles. [e.g., a central flattening of $p(r)$, if $q \sim 1$ on axis]. Much more serious, presumably even "disruptive", consequences would be observed in the case of overlap between singular reglons corresponding to different values of $q$. Such possibllities have encouraged theoretical and experimental interest in the tearing mode.

To avoid misunderstanding, we remark here that the linear analysis of tearing, to be considered in Sec. IV, differs from Eqs. (2)-(6), in that singular perturbations do not occur. In fact the cruclal feature of every tearing analysis consists of tinding and lincluding small corrections to the operator $\underset{\sim}{\mathrm{B}} \cdot \nabla$, and thereby resolving the singularity in equations analogous to Eq. (4). Of course our comments conceming Eq. (6) remain applicable, so long as the corrections are Indeed small.

\section{B. Observations on tokamaks}

Magnetic probe measurements on a number of tokamak plasmas have revealed sinusoidally oscillating magnetic field perturbations, with the spatial structure, $7,8,33$

$$
\widetilde{B} \propto \exp (i \mathrm{~m} \theta-\varphi), \quad m=1,2,3, \ldots
$$

By comparing the times of onset of such "Mirnov oscillations" with the time-evolution of the safety factor $q(t, t)$, it has been confirmed that a particular mode number $m$ occurs only when $q$ achieves the value of $m$ at some radius inside the plasma. 'This behavior coincides with that of 
both tearing modes and Ideal internal kink modes, although the former seem more likely to be unstable. ${ }^{9}$

The frequency of the Mirnov oscillations is usually Identifled with the electron diamagnetic frequency, the growth rate being typically smaller. This fact is also consistent with modern theories of the tearing mode.

Some cxperiments indicate an assoclation between Mirnov osclllations and local flattening of the electron temperature profile, near the approprlate rational surface $r_{s} \cdot{ }^{34}$ (This effect is most clearly visible for mores with $m \geq 2$. A similar flattening of, for example, the plasma density, is not ruled out, but more difficult to detect.) Since proflle-flattening is an obvious consequence of local flux surface destruction, these observations are especially suggestive.

Mirnov oscillations are relatively harmless by themselves. Howe ver, they frequently appear as precursors, elther to internal. "mini-disruptions", manifested in saw-tooth osclllations of the $x$-ray-detected electron temperature, or to major disruptions, resulting in abrupt collapse of the discharge ("disruptive instabllity"). Although patently nonlinear, both kinds of disruption have explained in terms of magnetic field line tearing. For example, nonlinear growth and decay of magnetic islands could be assoclated with mini-discuptions, $21,22,25$ while the major disruption might involve sudden overlap of islands growing from initialiy distinct rational flux surfaces. 25,26

It should be emphasized that all explanations of Mirnov oscillations and disruptions remain tentative, and several theories of the phenomena have been proposed which make no reference to the tearing mechanism. For example, the oscillations have been interpreted in terms of nelghboring helical equilibria, ${ }^{13}$ and in terms of temperature-gradient driven drift waves ${ }^{35}$ 
profile flattening near the magnetic axis (for $q=m=1$ ) has been explained in terms of a rotational interchange mode; ${ }^{36}$ and a modified neoclassical theory has been shown to predict disruptive effects, simflar to those which are observed. ${ }^{37}$ If tearing theories seem prominent among the various candidates for explaining Mirnov phenomena, it is probably because such theories hope to relate all the observations to a single, relatively simple idea: changes in the topology of the magnetic fleld.

Finally, we point out that the great wealth of detalled experimental information on Mirnov oscillations and disruptions has been barely touched upon here. It is known, for example, that the sawtooth signal changes phase across the mode-rational flux surface, that the major disruption is accompanied by a negative voltage spike, and so on. Thus any proposed theoretical picture of the oscillations and disruptions is subject to an unusually rich assortment of experimental tests. Since a successful theory of the Mirnov phenomena would bear on several questions ciucial to the performance of a tokamak reactor (such as transport scaling laws, or the previously mentioned question of $\dot{\beta}$ limitarions), Llit epportunity offered to theorists is clear. 


\section{Tearing Instability Mechanism}

A. Magnetic Shear

The importance of magnetic shear with regard to flux surface destruction has already been noted. In addition, conventional tearing instabllities are driven by the magnetic fleld energy associated with shear. Hence we begin by considering an unperturbed field $\sim_{0}(r)$ whose direction depends upon radius. Then an interior radius, $r_{\star}$, and a fixed unit vector $\hat{n}$ can always be chosen such that the field component $\underset{\sim}{B}=\hat{n}\left({\underset{\sim}{O}}_{0} \cdot A\right)$ changes $\operatorname{sign}$ at $r_{s}$,

$$
\stackrel{B}{O}_{0}\left(r_{*}\right) \cdot \hat{n}=0 \text {. }
$$

The lines of the $\underset{\sim}{B}$-field, in a radial neighborhood of $r_{*}$, are depicted in. Fig. 2 .

The minimum field energy configuration (vacuum field) is roughly characterized by the absence of shear:

$$
\underset{\sim}{B} \cdot \hat{n}=0, \text { for all } r \text {. }
$$

The configuration of Fig. 2 can relax to this minimum energy state by means of field annihilation: field lines above (below) $r_{\star}$ migrate downwards (upwards) so as to annihilate their oppositely directed counterparts. It is instructive to distinguish two mechanisms available for such migration: convection and diffusion.

These mechanisms are clearly visible in a resistive MHD model, in which we assume

$$
\underset{\sim}{E}+\underset{\sim}{V} \times \underset{\sim}{B} / \mathrm{C}=\eta \underset{\sim}{J}, \nabla \cdot \underset{\sim}{\mathrm{V}}=0,
$$

where $\eta$ is the (spatially constant) resistivity, $\mathcal{J}$ is the plasma current, $\underset{\sim}{\sim}$ the flow velocity and $\underset{\sim}{\mathrm{E}}$ the electric field. In slab geometry, Max- 
well's equations then yield the familiar linearized relation

$$
\frac{\mathrm{dB}}{\mathrm{dt}}=-\frac{\mathrm{a}^{2}}{\tau_{\mathrm{s}}} \nabla^{2} \underset{\sim}{\mathrm{B}}+i k_{\|} B_{\mathrm{O}} \underset{\sim}{\mathrm{V}}
$$

where $a$ is the plasma radius, $k_{\|}$is the parallel wave vector and

$$
\tau_{s} \equiv 4 \pi a^{2} / c^{2} \eta
$$

is the resistive skin-time. For a nominal ordering we have

$$
\nabla \sim a^{-1} \sim k_{\|}
$$

and $V \sim v_{A} \equiv\left(B_{0}{ }^{2} / 4 \pi \rho\right)^{\frac{1}{2}}$, the Alfvén speed. Then the first, diffusive term on the right-hand side of Eq. (12) corresponds to the time scale $\tau_{s} \sim 10^{-1}$ sec., while the second, convective term corresponds to the scale $\tau_{A} \approx a / \dot{v}_{A} \sim 10^{-8}$ sec. (These numerical estimates correspond very roughly to typical tokamak parameters.) Thus equilibrium magnetic field diffusion, characterized by $\nabla B \sim B / a$, is extremely slow, and significantly rapid field annihilation must involve the convective term.

However, a crucial property of ideal $\left(\tau_{s} \rightarrow \infty\right)$ MHD convection must be recalled: if $\xi$ is the plasma displacement, then the radial field perturbation $B_{r}$ satisfies

$$
\mathrm{B}_{\mathrm{r}} / \mathrm{B}_{\mathrm{O}}=1 \mathrm{k}{ }_{\|} \xi_{\mathrm{r}} \text {. }
$$

This can be seen from Eq. (12) or, more persplcuously, from Fig. 3; the essential point is that the field is "frozen" to the plasma fluid, in the ideal case.

B. Annihilation and reconnection

With these remarks in mind, we return to Fig. 2 , and consider how we would perturb this field to most quickly relax the shear. To produce rapid, Alfvénic excitation, we would "pluck" the rubber-band-like ${\underset{\sim}{n}}_{n}$-lines periodically along $\hat{n}$; to produce field annihilation, we must pluck them 
anti-symmetrically with respect to the radlus $r_{\star}$, so that a fleld line below $r_{\star}$ is drawn up at the same positions, along the $\hat{n}$-direction, that the corresponding field line above $r_{\star}$ is drawn down. The resulting kink-like perturbation is depicted in Fig. 4. Note that annihilation and reconnection, or "tearing", at $r_{*}$ has altered the magnetic field topology: magnetic islands have appeared. It is clear that this topological change occurs even for an infinitesimal perturbation, 1.e., even in linear theory, so long as the perturbation has the geometry we have described.

A simple characterization of this geometry is also clear from Fig. 4: the wave vector $\underset{\sim}{k}$ lies along the direction $\hat{n}$. It follows that the field component $\underset{\sim}{B}$ is proportional to $k_{\|}$, whence

$$
k_{\|}\left(r_{*}\right)=0 \text {, }
$$

by the definition of $r_{\star *}$. In an axisymmetric torus we must have $\underset{\sim}{k}=\hat{\theta} \mathrm{m} / \mathrm{r}-$ $\hat{\varphi} \mathrm{n} / \mathrm{R}$, where $\hat{\theta}$ and $\hat{\varphi}$ are the opvious unit vectors, $R$ is the major toroidal radius, and $\mathrm{m}$ and $\mathrm{n}$ are the poloidal and toroidal mode numbers respectively, so that Eq. (16) imples

$$
\mathrm{q}\left(\mathrm{r}_{\star}\right)=\mathrm{m} / \mathrm{n} \text {. }
$$

Thus the radius, $r_{*}$, must be identified with one of the radl1 $r_{S}$ of Eq. (6), if the tearing mechanism is to function.

A crucial conclusion follows from this identification and from inspection of Fig. 4: the tearing mode requires

$$
B_{r}\left(r_{s}\right) \neq 0
$$

in contradiction to Eq. (15). In other words, significant decoupling of the plasma fluid and the perturbed magnetic field lines must occur at the rational flux surface. Of course, the decoupling need not in general result from resistivity. 
It is clear that the departure from ideal behavior described by Eq. (17) is only locally significant: at most radil $r \neq r_{s}$, the second term of Eq. (12) easily dominates the first. Thus the tearing mode appears as a kink-instability, slightly modified near rational surfaces. For a complementary description, emphasizing diffusion, we may consider the narrow region near $r_{s}$ '

$$
\left|r-r_{s}\right| \leq \lambda<a
$$

in which Eq. (14) does not pertain, and the two terms on the right-hand side of Eq. (12) are comparable. In this "tearing layer", one expects (from, for example, Fig. 4) that $\lambda$ would replace a as a measure of the radial scale length:

$$
\frac{\mathrm{a}^{2}}{\tau_{\mathrm{s}}} \nabla^{2} \underset{\sim}{\sim} \sim\left(\frac{\mathrm{a}}{\lambda}\right)^{2} \underset{\mathrm{T}_{\mathrm{s}}}{\stackrel{\mathrm{B}}{\sim}} \gg \mathrm{B} / \mathrm{T}_{\mathrm{S}}
$$

Detailed analysis, to be outlined in the following Section, reveals a somewhat more complicated situation; in many cases only one factor of $(a / \lambda)$ actually appears, and the skin time ${ }^{\mathrm{s}}$ need not always be resistive. But the essential point is generally valld: enhanced radlal gradients in the tearing layer yield relatively rapid, localized diffusion of the perturbed fleld. By identifying such diffusion with the tearing instability, we can correctly estimate its growth rate, and we omit only its non-local, kink-like, "wake".

In summary, the tearing instability takes advantage of field-line tension, through the Alfvén mechanism, to allow shear-relaxation to proceed on time scales much shorter than the skin time. The instability can occur only in the presence of rational flux surfaces, in the close vicinity of which the (small) equilibrium field component $B_{n} \simeq B_{\theta}(1-n q / m)$ is annihilated and reconnected. The tearing of $B_{n}$-lines requires non-ideal behavior, $B_{r}\left(r_{s}\right) \neq 0$, and can be considered analogous to localized magnetic field 
diffusion. But the tearing mode itself is not localized: it is a convectiondiffusion hybrid which appears, far from the rational surface, similar to the kink mode. 


\section{Linear analysis of tearing instabilities}

\section{A. Boundary Layer Problem}

The previous two Sections indicate the importance of modes having the following properties:

(i) a kink-like structure, far from the rational flux-surface (i.e., for $\left.k_{\|} \sim a^{-1}\right)$;

(ii) $\mathrm{B}_{\mathrm{r}}\left(\mathrm{r}_{\mathrm{s}}\right) \neq 0$;

(iii) low frequency: $\omega$ or $Y \equiv \operatorname{Im}(\omega)$ should be small compared to $k_{\perp} v_{A}$, as in the observed tokamak oscillations;

(iv) $Y>0$ in parameter ranges of interest to present or planned tokamak experiments. It seems convenient to identify any mode having properties (1) and (1i) as a tearing mode; with this definition, most known tearing modes have been found to satisfy property (iii). Property (Iv) is more dellcate, Ir! linat the stability or consistency criteria for a given, theoretically proposed, tearing mode often depend strongly on fine details of the equilibrium. We shall not use property (iv) restrictively here.

We begin our outline of the linear analysis by considering the kinked region, exterior to the tearing layer. Here, the slow plasma motion indicated by property (iii) allows us to neglect, as a first approximation. inertial and viscous effects in the equation of motion. Thus we consider a perturbed equilibrium, with approximately scalar pressure:

$$
c \nabla \tilde{\mathrm{p}}=\tilde{\sim} \times \mathrm{B}_{0}+\sim_{0} \times \underset{\sim}{\widetilde{B}} \text {. }
$$

Equation (20), together with the Maxwell equations

$$
\nabla \cdot \underset{\sim}{B}=0, \nabla \times \underset{\sim}{B}=4 \pi J / C,
$$


provides a closed, homogeneous system for the seven unknown quantities $\widetilde{\mathrm{p}}, \underset{\sim}{\sim}$ and $\underset{\sim}{\sim}$. In cylindrical geometry, the system may algebraically be reduced to a second order differential equation which involves only the radial field perturbation. This is the so-called Newcomb equation: ${ }^{38}$

$$
k_{\|}\left(r \psi^{\prime} / k^{2}\right)^{\prime}-\left(\psi / B_{0}\right)\left[r\left(k_{\|} B_{0}\right)^{\prime} / k^{2}\right]^{\prime}-r g \psi=0,
$$

with

$$
g \equiv k_{\|} \frac{k^{2} r^{2}-1}{k^{2} r^{2}}-\frac{2 n^{2}}{k^{2} r^{2}}\left(\frac{r}{R}\right)^{2}\left[\frac{k_{1}}{k^{2} r^{2}}-\frac{4 \pi p_{o}}{B_{o}^{2}}\right] \text {. }
$$

Here,

$$
\psi=-i \tilde{B}_{r}
$$

is the conventional measure of the field perturbation (or axial vector potential), the primes denote radial derivatives, and the wave vector components are given by

$$
\begin{aligned}
& k_{\|}(r)=\frac{m}{r} \frac{B_{\theta}}{B_{O}}-\frac{n}{R} \frac{B_{z}}{B_{O}} . \\
& k_{\perp}(r)=\frac{m}{r} \frac{B_{z}}{B_{0}}+\frac{n}{R} \frac{B_{\theta}}{B_{O}} .
\end{aligned}
$$

whence

$$
k^{2} r^{2}=m^{2}+(r / R)^{2} n^{2}
$$

with $(m, n)=1,2,3, \ldots$. Axial perlodicity is assumed; so that the cylinder models a torus with major radius $R$.

The first noteworthy feature of Eq. (21) concerns its derivation: such standard assumptions of ideal MHD as

$$
\begin{aligned}
& \underset{\sim}{\mathrm{E}}+\underset{\sim}{\mathrm{V}} \times \underset{\sim}{\mathrm{B} / \mathrm{c}=0,} \\
& \mathrm{p} \rho^{-\gamma}=\text { const. . } .
\end{aligned}
$$

and so on, have not been used, and need not pertain. The question of incompressibility is similarly irrelevant. The fact that Eq. (21), which 
is conventionally derived from the MHD energy principle, does not in fact depend upon MHD assumptions, is significant, since the observed oscillations seem too slow to be correctly described by an MHD model.

Of course, the most important feature of the Newcomb equation is that $k_{\|}$, which appears in the coefficient of the highest order, $\psi "$, term, may in general vanish at some radius $r_{s}$

$$
k_{\|}\left(r_{s}\right)=0 \text {. }
$$

If Eq. (21) is presumed valid in the close vicinity of $r_{s}$, then any acceptable solution $\psi$ must satisfy

$$
\psi\left(r_{s}\right)=0
$$

in contradiction to property (ii). Examples of modes satisfying Eqs. (21) and (28) are the local, Suydam modes, and the non-local, 1deal-MHD kink modes (which are referred to as "internal" or "external", depending upon whether $\mathrm{r}_{\mathrm{s}}$ lies in the plasma, or in a vacuum region surrounding the plasma, respectively. ${ }^{9}$ ) The relation between the existence of such solutions and MHD stability was studied in detail by Newcomb. ${ }^{38}$

More generally, and more realistically, we must acknowledge that Eq. (21) may be a poor approximation in the region close to $r_{S}$. The description of this region by a more accurate differential equation, which is not singular at $r_{S}$, leads to the concept of a "tearing layer", as des cribed in Section III.

To anticlpate salient features of the tearing layer equation, note that it is $\psi^{\prime \prime} \sim \psi / k_{\|}$, rather than $\psi$ itself, which becomes large as $r$ approaches $r_{s}$. To "turn-over" $\psi$ " - to round off the tip of its spike at $r_{s}$ - a curvature term of the form $\left(\psi^{\prime \prime}\right) " \sim \psi^{\prime \prime} / \lambda^{2}$, where $\lambda$ is the layer width of Eq. (18), is 
required. Such a term will presumably come from including, inter alia, $\omega / k_{\perp} \cdot v_{A}$ corrections to the force balance equation. On the other hand, terms in this equation which are small in $\lambda / a$ may be omitted. Thus we expect the tearing layer to be described in general by an equation of the form

$$
\psi " \prime+\alpha(\omega) h(r)(\psi "+\ldots)=0(\lambda / a)
$$

The final step in the linear analysis of tearing instabilities consists of treating Eqs. (21) and (29) as a standard boundary layer problem: one seeks the regular solutions of Eq. (29) which smoothly join, as $\left|r-r_{s}\right|$ becomes large, onto the corresponding solutions of Eq. (21). Of course the latter must also satisfy boundary conditions at $r=0$ and $r=a$. Applied to Eq. (29), the regularity and "matching conditions" in general restrict $\alpha(\omega)$ to a discrete set of values,

$$
\alpha(\omega)=\alpha_{j}, j=1,2, \ldots
$$

where the $\alpha_{j}$ typically depend upon asymptotic properties, for $r \rightarrow r_{S}$ ' of the exterior solutions, i.e., the solutions to Eq. (21).

Equation (30) represents the tearing mode dispersion relation. We next turn our attention to a concrete example.

B. Classical tearing mode

Noting that Eq. (20) imples Eq. (1), and recalling that Eq. (1) leads to singular perturbations, $\widetilde{p} \propto\left(k_{\|}\right)^{-1}$, we conclude that small corrections to Eq. (20) will be important when $k_{\|}$is small. The simplest such correction comes from inertia:

$$
\rho \underset{\sim}{V} / \partial t=-\rho \omega^{2} \underset{\sim}{\xi} .
$$


where $\underset{\xi}{\xi}$ is the plasma displacement, and $\rho$ the equilibrlum mass-density. We add the inertial term to Eq. (20), which is then used to compute the perturbed current perpendicular to $\underset{\sim}{\mathrm{B}}$. As a result we find

$$
\tilde{J}_{r}=\left(c / B_{0}\right) \cdot\left(\rho \omega^{2} \xi_{\perp}-1 k_{\perp} \tilde{p}\right)
$$

where $\xi_{\perp} \equiv\left(\mathbb{\sim}_{O} \times \hat{r} / B_{O}\right) \cdot \underset{\sim}{\xi}$ and $k_{\perp} \equiv\left({\underset{\sim}{O}}_{0} \times \hat{r} / B_{O}\right) \cdot \underset{\sim}{k}$, as in Eq. (24). Next , we approximate for $\lambda / a<1$, by retaining only the highest order radial derivatives of perturbed quantities. Then, since $\underset{\sim}{\nabla} \cdot \underset{\sim}{\sim}=0^{\circ}$, Ampere's law has the parallel component

$$
4 \pi \tilde{J}_{\|} / c \cong \psi^{\prime \prime} / k_{\perp}
$$

and the quasineutrality condition,

$$
0=\underset{\sim}{\nabla} \cdot I \cong J_{r}^{\prime}+i k_{\|} J_{\|}
$$

takes the form

$$
k_{\|} B_{O} \psi^{\prime \prime}+4 \pi \rho \omega^{2}\left(i k, \xi_{\perp}\right)^{\prime} \cong 0 \text {. }
$$

Here we have omitted a contribution from the $\tilde{\mathrm{p}}$-term $\ln \tilde{\mathrm{J}}_{\mathrm{r}}$, using $\widetilde{p} \cong-\xi_{r} p_{0}^{\prime}$ and $\xi_{r} / \xi_{\perp}=0(\lambda / a)$, as will be seen presently. We note, however, that the pressure perturbation is Important for certain low-frequency, drift-tearing modes, considered in Sec. V. It can also affect the classical tearing mode, when toroidicity is included. Physically, Eq. (31) states that the crucial accelerating force is field-line tension, $\underset{\sim}{\left.(\mathbb{D}: \nabla)_{r}\right)} \sim k_{\|} B_{0} B_{r}$. in qualitative agreement with the discussion of Sec. III.

Note that the first term of Eq. (31) is simply the highest-order derivative term of the Newcomb equation. The second term in Eq. (31) wlll be seen to resolve the singularity at $r_{s}$, after we have obtained an equation for $\underset{\sim}{\xi}$ : 
The classical prescription for $\underline{\xi}$ is provided by Ohm's law and incompressibility: Eqs. (11). Thus the radial component of Eq. (12) relates $\xi_{r}$ to $\psi$, while

$$
\nabla \cdot \underset{\sim}{\xi}=0 \approx i k_{\perp} \xi_{\perp}+\xi_{r}^{\prime}+0(\lambda / a)
$$

allows Eq. (31) also to be written in terms of $\psi$ and $\xi_{r}$. We then obtain the equations ${ }^{2}$

$$
\begin{aligned}
& k_{\|} B_{O} \psi^{\prime \prime}-4 \pi \rho{ }^{2} \xi_{r} "=0 \\
& \left(a^{2} / \tau_{s}\right) \psi "+i \omega\left(\psi-k_{\|} B_{O} \xi_{r}\right)=0
\end{aligned}
$$

which evidently may be combined to yield a fourth order differential equation, 
the tearing layer equation of Eq. (29), for $\psi$.

Note that the second term of Eq. (33) is proportional to the parallel electric field,

$$
\mathrm{i}_{\omega}\left(\psi-\mathrm{k}_{\|} \mathrm{B}_{\mathrm{O}} \xi_{\mathrm{r}}\right) \simeq \mathrm{ck_{ \perp }} \mathrm{E}_{\|} .
$$

if a small term, $\mathrm{c} \eta \mathrm{k}_{\|} \mathrm{J}_{\perp}$, is omitted. For ordinary resistive diffusion, $\psi^{\prime \prime} \sim \psi / a^{2}$, and Eq. (33) states that $E_{\|} \sim \psi / c k_{\perp}{ }_{s}$ is very small, as in ideal MHD. Thus the tearing layer may be characterized as that region in which $E_{\|}$significantly exceeds its resistive diffusion value; recall Eq. (19).

Because we have omitted all finite Larmor radius effects, and because the simple form of Ohm's law we have used applles only for large collision frequency, $\nu$,Eqs. (32) and (33) have a limited range of validity. In particular they require

$$
\nu \gg \omega \gg \omega_{\star},
$$

where $\omega_{*}$ is a diamagnetic frequency. It is significant that the three frequencies appearing in Eq. (35) are in fact roughly comparable in many present tokamak experiments. On the other hand, several more recent studies, which allow $\omega \sim \omega_{*}$ and $\omega \sim \nu$, yield tearing layer equations nearly identical in form to Eqs. (32) and (33); only the coefficients are changed. We therefore regard the equations as archetypal, and consider next some properties of their solution.

\section{Matching Condition}

To es timate the growth rate, $\gamma=-1 \omega$, and layer width, $\lambda$, of the classical tearing mode, we assume both terms in each of Eqs. (32) and (33) are comparable, and expand

$$
\begin{aligned}
k_{\| 1} & \approx k_{\| \prime}\left(r-r_{s}\right) \\
& \sim k_{\|}^{\prime} \lambda .
\end{aligned}
$$


In the most obvious ordering, we would further estimate $\xi_{r}{ }^{\prime \prime} \sim \xi_{r} / \lambda^{2}$ and

$$
\psi " \sim / \lambda^{2}
$$

Then Eq. (33) yields

$$
Y \sim(a / \lambda)^{2} \tau_{s}^{-1}
$$

corresponding to localized diffusion, while Eq. (32) yields

$$
Y \sim k_{\|} v_{A} \sim k_{\|}^{\prime} \lambda v_{A} \text {. }
$$

corresponding to Alfvén convection ( $v_{A}$ is the Alfvén speed). After eliminating $\lambda$ we find, ${ }^{39}$ with $\tau_{A} \equiv a / v_{A}$.

$$
\gamma^{3} \sim\left(k_{\|}{ }^{\prime} a^{2}\right)^{2} /\left(\tau_{s} \tau_{A}^{2}\right),
$$

a result which confirms several tearing mode features anticipated in Sec. III: the instability is driven by shear, and has the form of a diffusion-convection hybrid.

However, Eqs. (36) and (37) do not describe the tearing modes most frequently considered in the literature. The point is that, unless $\psi\left(r_{s}\right)$ is small, Eq. (36) corresponds to an apparent (1.e., when vlewed with respect to the scale length $a \gg \lambda$ ) discontinuity in the radial fleld perturbation. It can be seen from Fig. 4 that such a discontinuity is not required, nor even helpful, for the tearing instabllity mechanism to operate. In a less extreme case, $\psi$ itself is continuous, and only its slope, $\psi^{\prime}$, changes appreciably across the tearing layer. The apparent discontinuity in slope is measured by

$$
\Delta^{\prime} \equiv\left[\psi^{\prime}\left(r_{s}+\epsilon\right)-\psi^{\prime}\left(r_{s}-\epsilon\right)\right] / \psi\left(r_{s}\right)
$$

where $\lambda \ll \varepsilon \ll a$, and Eq: (36) becomes in this case

$$
\psi " \sim \Delta \psi\left(r_{s}\right) / \lambda \text {. }
$$

The nominal ordering, $\Delta^{\prime} a \sim 1$, 'implies that $\psi(r) \sim \psi\left(r_{S}\right)$ throughout the 
tearing layer ${ }^{2}$ this "constant- $\psi$ approximation" is frequently used, though occasionally disputed, to simplify the solutions of Eq. (32) and (33).

Without making any assumption regarding the magnitude of $\Delta^{\prime}$, we may repeat the manipulations leading to Eq. (37), but now us ing Eq. (39) instead of Eq. (36). Then Eq. (33) implies

$$
Y \sim \frac{a^{2} \Delta^{\prime}}{\tau_{s}{ }^{\lambda}}
$$

while Eq. (32) yields $k_{\|} B_{O} \Delta^{\prime} \psi / \lambda \sim 4 \pi \rho \gamma^{2} \xi_{r} " \sim\left(B_{O} / v_{A}\right)^{2} \gamma^{2} \varepsilon_{r} / \lambda^{2}$. Assuming that the two terms on the left-hand side of Eq. (34) contribute comparably to $E_{\|}$, we obtain

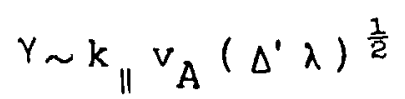

We can solve Eqs. $(40)$ and (41) for $Y$ and $\lambda$ in the two cases of interest: (i) If $\Delta^{\prime} \sim a^{-1}$, we find

$$
\gamma^{5} \sim\left(k_{\|} a^{2}\right)^{2}\left(\Delta^{\prime} a\right)^{4} \tau_{s}{ }^{-3} \tau_{A}^{-2}
$$

where instability occurs only if $\Delta^{\prime}>0$. The width is given by

$$
(\lambda / a)^{3}=\left(\Delta^{\prime} a\right)\left(k_{\|}^{\prime} a^{2}\right)^{-2}\left(\tau_{A} / r_{S}\right)^{2}
$$

A typical solution $\psi(r)$ for this case is depicted in Fig. 5. The growth rate of Eq. (42) is typically smaller than that of Eq. (37); yet it also displays the shear-driven, hybrid features which were noted prevlously. (ii) If $\Delta^{\prime} \sim \lambda^{-1}$, we obviously recover Eq. (37) for $\gamma$. The width in this case satisfies

$$
(\lambda / a)^{3} \sim\left(k_{\|} a^{2}\right)\left(\tau_{s} / \tau_{A}\right)
$$

Two quite different radial mode structures can produce $\Delta^{\prime} \sim \lambda^{-1}$, and thus Eqs. (37) and (44). First, $\psi\left(r_{s}\right)$ might be quite small, of order $\mathrm{Na}$ compared to its maximum value outside the layer. This circumstance is 
depicted in Fig. 6a; we shall see that it commonly applies to modes with $\mathrm{m}=\mathrm{n}=1$. Second, it could happen that $\psi^{:}$is large, proportional to $\lambda^{-1}$, near $r_{S}$. Then $\psi$ would appear nearly discontinuous, as shown in Fig. $6 \mathrm{~b}$. This possibility is rarely considered in the literature, although both analytical 40 and numerical ${ }^{23}$ studies have indicated its possible importance.

It should now be clear that analytical solution of the tearing layer equations is not possible without some a priori knowledge of the behavior of $\psi$ outside the tearing layer: Eqs. (21), (32) and (33) must in general be solved simultaneously. In most of the literature, this task is avoided by assuming $\psi$ to be continuous across the layer, as in case (i). It is then reasonable to consider the tearing mode problem solved, once $Y$ has been evaluated in terms of $\Delta^{\prime}$.

As noted previously,. case (ii) is of importance mainly for $m=n=1$ modes . The distinguishing feature of such modes is apparent from Eqs. (22) and (25), which show that

$$
g \sim(n r / R)^{2} \ll 1 \text {, for } m=1 .
$$

Since toroidal curvature terms, which Eq. (21) omits, are also of order $\left(r^{2} / R^{2}\right)$, the use of a cylindrical model for $m=1$ modes is questionable. ${ }^{11}$ But even in a torus, a lowest order approximation for the $\mathrm{m}=1$ internal kink mode presumably would be obtained by neglecting $g$, so that. Eq. (21) is approximated by

$$
k_{\|} B_{O}\left(r \psi^{\prime} / k^{2}\right)^{\prime}=\left[r\left(k_{\|} B_{O}\right)^{\prime} / k^{2}\right] \psi .
$$

or

$$
\left[r\left(k_{\|} B_{o}\right)^{2}\left(\psi / k_{\|} B_{o}\right)^{\prime} / k^{2}\right]^{\prime} \cong 0
$$

Thus the exterior solution is characterized by ${ }^{12}$

$$
\psi / k_{\|} B_{0} \sim \xi=\text { const. , for } r<r_{s}
$$


and $\psi / k_{\|} B_{O}=0$ for $r>r_{S}$, to satisfy the boundary condition at $r=a$ ("fixed-boundary" kink mode). It is clear that this form of the exterior solution corresponds to Fig. 6a: $\psi \approx$ const. $k_{\|} \lambda$, for $r \sim r_{s}$.

The preceding argument has sometimes been taken to imply a strict correspondence between $\mathrm{m}=1$ modes and Eq. (37), on the one hand, and between $\mathrm{m} \geq 2$ modes and Eq. (42), on the other. In fact the correspondence is far from clear-cut; in particular, it is contradicted by $\mathrm{m} \geq 2$ modes having the discontinuous form depicted in Fig. $6 \mathrm{~b}$. Furthermore, $\mathrm{m}=1$ modes have been found whose growth tales resiemble Eq. (42). 39

Once the influence of the form of the exterior solutions is understood, the analytical solution of Eqs. (32) and (33) is relatively stralghtforward. An especially elegant procedure is presented in Ref. 39. We do not review the detailed solution here.

D. Summary

To recapitulate: tearing instabilities are analyzed by solving a boundary layer problem for the mode-rational flux surface. The kink-like excitation of the exterior region is sufficiently slow to be treated as a neighboring equilibrium. With the neglect of stress anisotropy, this description corresponds to the marginal stability condition of ideal MHD (Newcomb equation). In the interior region, $B_{r}{ }^{\prime \prime}(r)$ becomes large, and the description. hinges upon coupling $\mathrm{B}_{\mathrm{r}}$ " to the parallel electric field of Eq. (34). Various nominally small effects, of which only resistivity has been considered here, can provide the coupling. The resulting tearing layer equation is generally (equivalent to) a fourth order radial differential equation for ${ }^{\prime} B_{r}$. The form of the final dispersion relation depends not only upon the $B_{r} "-E_{\|}$ coupling, but also upon gross features (e.g., continuity across the layer) 
of the exterior solutions. Similarly, the precise value and sign of the growth rate can depend upon critical detalls (e.g.. $\Delta^{\prime}$ ) of the exterior solutions.

The coupling between $B_{r}$ " and. $E_{\|}$becomes particularly delicate at low frequency and small resistivity. When $\omega \sim \omega_{*}$ as for the observed modes, a host of higher order terms; in both the equation of motion and the (generalized) Ohm's law, become potentially important. This explains why, fourteen years after the lengthy FKR study, the linear theory of tearing instabilities remains of interest. It also explains how the analys is outlined above can yield a remarkably disparate collection of dispersion relations, as shown in the following section. 


\section{Tearing Mode Dispersion Relations}

Finally we display some tearing mode growth rates. What follows is at best a representative sampling, and far from complete. Our purpose is not only to display the wide variety of results whlch can be obtalned, but also to touch upon some topics of recent interest.

Future research on tearlng modes may. well be concentrated on their nonlinear properties. Yet a characteristic feature of the modes - their sensitive dependence on small detalls - suggests a long-term interest in the linear theory.

A. Classical tearing modes

The FKR tearing mode growth rate; derlved from a slab model with $\nu>v \gg \omega_{*}$, is given by ${ }^{2}$

$$
v=y_{c} \equiv\left\{\left[\frac{\Delta^{\prime} a \Gamma\left(\frac{1}{4}\right)}{2 \pi \Gamma(3 / 4)}\right]^{4} \frac{\left(k_{\|}^{\prime} a^{2}\right)^{2}}{{ }_{\tau_{s}{ }^{3}{ }^{2}{ }^{2}}{ }^{1 / 5}}\right\}^{1 / 5}
$$

for $\Delta^{\prime} a \sim 1$, as in case (1) of Sec. IV. Here, $\Delta^{\prime}$ is given by Eq. (38), $\tau_{s}$ is the resistive skin time of Eq. (13) and $\tau_{A}=a / v_{A}$ is the Alfven time. Of course, this result is just the exact version of Eq. (42). We recall that instability occurs only when $\Lambda^{\prime}>0$.

Equation (45) describes a resistive tearing mode: the decoupling of plasma and magnetic field which allows $B_{r}\left(r_{s}\right) \neq 0$ is provided by resistivity. As noted previously, other miechanisms can be similarly effective. In the low collision frequency limit, the Inertial term, $4 \pi u{ }_{p}^{-2} \partial J / a t$, takes the place of $n \mathrm{~J}$, in Ohm's law. The resulting growth rate, 3,4

$$
\gamma=\left(8 \gamma_{c}^{5} \nu^{-3}\right)^{\frac{1}{2}} \text {. }
$$

may be obtained directly from Eq. (45), by the substitution $\nu \rightarrow 2 \gamma$ (the factor of 2 comes from the Spltzer-Harm 41 resistivity for unit lonic change). 
Equation (46) describes the classical collionless tearing mode. A distinct type of collisionless tearing mode, in which electron inertia is similarly responsible for field-fluid decoupling, is considered below.

The classical tearing mode instability criterion, $\Delta^{\prime}>0$, strictly pertains only in cylindrical or slab geometry. The same resistive fluid model which yields Eq. (45) has recently been analyzed in toroldal geometry. ${ }^{16}$ It is found that $\Delta^{\prime}$ should be replaced by a much more complicated quantity, $\Delta^{\prime}$ * (our notation), which includes in particular the effects of toroldal curvature. More surprising is the discovery that, under certain circumstances, the difference between $\Delta^{\prime}$ and $\Delta^{\prime}$ * can be significantly large.

B. Drift-tearing modes

"Drift-tearing" modes have frequencies comparable to the diamagnetic frequency, $\omega_{*}$. In this case, the Ion dlamagnetic drift enters the equation of motion, through perturbation of the lon pressure (magnetoviscous contributions are cancelled by the convective inertial term). ${ }^{6,42}$ One finds that Eq. (32) should be replaced by

$$
4 \pi \rho\left(\omega-\omega_{i \star}\right) \xi "-k_{\|} R_{0} \psi^{\prime \prime}=0 .
$$

where $\omega_{i *}=\left(\mathrm{cm} / e B_{0} r\right) p_{i} / n_{i}, m=1,2, \ldots$. Similarly, perturbation of $p_{e}$ yields important $\omega_{e^{*}}=-\left(\mathrm{cm} / e \mathrm{~B}_{\mathrm{o}} \mathrm{r}\right) \mathrm{p}_{e^{1 / \mathrm{n}_{e}}}$ terms in the Ohm's law of Eq. (33). The simplest version of the resulting dispersion relation may be written as 4,13

$$
\left.\omega\left(\omega-\omega_{i *}\right)(\omega)-\omega_{e *}\right)^{3}=i \gamma_{C}{ }^{5} .
$$

Equation (47) pertains to an isothermal plasma, and, strictly speaking, requires $\nu \gg w$. The point is that the static, or "dc" resistivity in Ohm's law cannot describe perturbations with $\omega \sim \nu$. When an "ac" Ohm's law 
is used, and when electron temperature gradient effects are retained; one obtains the dispersion relation ${ }^{14}$

$$
\omega\left(\omega-\omega_{i^{*}}\right)\left(\omega-\omega_{e^{*}}-\alpha_{2} \omega_{T^{*}}\right)^{3} \alpha_{1}^{3}=1 \gamma_{C}{ }^{5}
$$

where the $\alpha^{\prime}$ s are complex-valued functions of $\omega$ and $\nu$, and $\omega_{T^{*}} \equiv$ - $\left(\mathrm{cm} / \mathrm{e} B_{0} r\right) T_{e} \cdot$. A variational solution to the guiding-center Fokker-Planck equation yields

$$
\begin{aligned}
& \alpha_{1}=0.98(1-0.54 i \omega \tau)\left(1-2.97 i \omega \tau-1.04 \omega^{2} \tau^{2}\right)^{-1}- \\
& \alpha_{2}=0.80(1-0.54 i \omega \tau)^{-1}
\end{aligned}
$$

where $\tau \sim \nu^{-1}$ is the electron collision time of Braginskil, ${ }^{43}$ for unit ionic charge:

$$
\tau=\frac{3}{16 \pi^{\frac{1}{2}}} \frac{m_{e}^{2} v_{T e}{ }^{3}}{e^{4} z^{2} n_{e} \ell n \Lambda}
$$

Equation (49) can be shown to include Eqs. (45)-(48) as limiting cases. It also predicts a new, "thermo-electric" tearing instability, which is driven, not by magnetic shear, but by the electron temperature gradient : 14,15

$$
Y=0.43\left(\omega_{e^{*}}+0.8 \omega_{T^{\star}}\right) \omega_{T^{*}} \text {, for } \omega_{\star}{ }^{\top}<1 .
$$

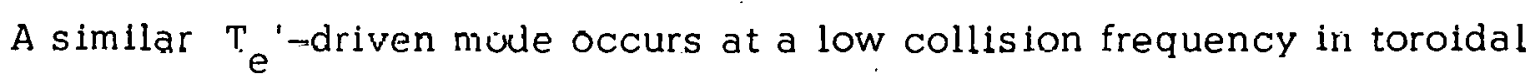
geometry, where magnetic trapping of electrons is important. ${ }^{17}$

C. Current channel modes

In the derivation of Eq. (48) or (49), terms of order $k_{\|} v_{T e}\left[v_{T e} \equiv\left(2 \mathrm{~T}_{\mathrm{c}} / \mathrm{m}_{\mathrm{e}}\right)^{\frac{1}{2}}\right]$ are presumed small compared to $\omega$ or $\nu$. Hence at small collision frequency, $\omega>>$, the relation between drift-tearing modes and electrostatic drift waves $\left(\omega<k_{\|} v_{T e}\right)$ is obscured. But the essential effects of finite $k_{\|} v_{T e} / \omega$ can be understood from a simple, isothermal electron fluld model. By retaining both the electron pressure gradient term and the inertial term in Ohm's law, we 
find that Eq. (33) should be replaced by 6,13

$$
\frac{a^{2}}{\tau_{s}} \psi^{\prime \prime}+i\left[\frac{\nu}{\nu-i \omega+i k_{\|}{ }^{2} v_{T e}{ }^{2} / \omega}\right]\left(\omega-\omega_{e^{*}}\right)\left(\psi-k_{\|} B_{0} \xi\right)=0
$$

Here some numerical factors are omitted for simplicity, and $\nu=\left(\omega_{p}{ }^{2} a^{2} / c^{2}\right) \tau_{s}^{-1}$. The familiar differential equation for drift waves is now obtained by using Eq. (47) to eliminate " $\psi$ " from Eq. (51), and then considering the limits $\omega \ll k_{\|} v_{\mathrm{Te}}$ (thus excluding the immediate vicinity of $r_{s}$ ), $\omega \gg \nu$. and $k_{\|} B_{0} \xi \gg \psi \psi$ (thus excluding coupling to an exterior, kinked region). Note that in this case $\xi$ cannot be interpreted as the plasma displacement; it strictly measures the electrostatic $\underset{\sim}{\mathrm{E}} \times \underset{\sim}{\mathrm{B}}$ drift.

More interestingly, Eq. (51) shows that for sufficiently small $\omega$ and,$\nu$, two nested singular regions can appear. Recall that the usual tearing layer is defined by the presence of an appreciable parallel electric field $\left(\psi-k_{\|} B_{0} \xi \neq 0\right)$. The width, $\lambda$, of this region may be estimated, for a given $\omega_{1}$ from Eq. (41). But the square-bracketted tactor $\mathrm{Ln} \mathrm{Eq}$. (51) can make " "become small, as one proceeds away from $r_{s}$, before the tearing layer bouridary ls reached. The resulting inner singular region evidently corresponds to a current channel (since $\left.\psi " \propto \mathrm{J}_{\|}\right) ;{ }^{15}$ denoting the current channel half-width by $\lambda_{C}$, we have, from Eq. (51),

$$
k_{\|}^{2} v_{T e}{ }^{2} \sim\left(k_{\|}{ }^{\prime}\right)^{2} v_{T e}{ }^{2} \lambda_{c}^{2} \sim|\omega(\nu-i \omega)|
$$

Our discussion in Sec. IV, and also Eqs. (45)-(49), assume $\lambda_{C} \geq \lambda$, so that the current channel is not distinguishable. In the opposite case,

$$
\lambda_{\mathrm{c}} \ll \lambda
$$

a somewhat different analysis pertains: in general; one must solve a double boundary layer problem, matching solutions for the current channel to those for the ordinary tearing layer, before matching the tearing layer solution to 
kink modes in exterior regions. ${ }^{17}$ We do not review solution to the double boundary layer problem here, beyond remarking that the current channel analysis itself is straightforward, because the lon motion, as in Eq. (32), is relatively unimportant.

In the simplest situation, the current channel solution can be matched directly to the exterior kink, without regard to the outer tearing layer. Notice that in this case, the quantity $\Delta^{\prime}$ of Eq. (38) must be defined with respect to $\lambda_{C}$, rather than $\lambda$. When $\nu<\omega$, so that Eq. (52) becomes

$$
\lambda_{C} \sim\left(\omega / k_{\|}^{\prime} v_{\underline{T} e}\right) .
$$

this procedure yields a collisionless tearing mode, alluded to previously, which is quite different from that of Eq. (46). The growth rate of this "current-channel collisionless tearing mode" is given by ${ }^{5}$

$$
\gamma=\gamma_{C C} \equiv \frac{\Delta^{\prime} k_{\| \prime}^{\prime}}{2 \pi^{\frac{1}{2}}}\left(\frac{c}{\omega_{p e}}\right)^{2} v_{\mathrm{Te}}
$$

For tokamak parameters, Eq. (54) indeed ylelds $\lambda_{C}<\lambda ; E q .(46)$ is not similarly consistent. 15

More recently, the same matching procedure has been applled to a collision dominated current channel. ${ }^{15}$. This will occur if $\nu>\omega$ but

$$
\lambda_{C} \sim\left(k_{\|}{ }^{\prime} v_{T e}\right)^{-1}(u v)^{\frac{1}{2}} \ll \lambda \text {. }
$$

as can be seen from Eq. (52). The resulting "semi-collisional" tearing mode has the growth rate

$$
\gamma=\left[3 \pi^{\frac{1}{4}} / 4 \Gamma(1 V / 4)\right] \gamma_{\mathrm{CC}}^{2 / 3} \cdot V^{\frac{1}{3}}
$$

for $Y \gg \omega_{\star}$, and a more complicated expression for the case $Y \ll \omega_{*}$.

A more transparent version of Eq. (53), the criterion for formation of a current channel, is difficult to construct. The problem is that $\lambda_{C} / \lambda$ depends not only upon $w$, which is usually not known in advance, but also, 
rather delicately, upon the shear, the plasma $\beta$, and so on. Thus the possible appearance of a current channel is best examined in individual cases.

D. $m=1$ modes

The classical $m=1$ tearing mode corresponds to case (ii) of Sec. IV B; that is, $\Delta^{\prime} \lambda \sim 1$, because $\psi\left(r_{s}\right)$ is small. In this case our previous growth rate estimate, Eq. (37), is fortuitously exact;

$$
\gamma=\gamma_{C l} \equiv\left(k_{\|} a^{2}\right)^{2} /\left(\tau_{S} \tau_{A}{ }^{2}\right)
$$

More recently the relation between tearing modes and ideal MHD kink modes, at $m=1$, has been examined. ${ }^{39}$ It is found that Eq. (37) pertains when the kink is marginally stable, and that when the kink is actually stable, $\gamma_{\mathrm{MHD}}<0$, a new type of tearing instabllity can appear. Disregarding diamagnetic effects, one obtains for this mode the growth rate

Here,

$$
Y=\left\{\left[\frac{\Gamma(5 / 4) r_{6}}{\pi \Gamma\left(-\frac{1}{4}\right) a G}\right]^{4} \frac{\left(k_{\| 1}^{\prime} a^{2}\right)^{6}}{T_{A}^{\prime 2} T_{S}{ }^{3}}\right\}^{1 / 5}
$$

$$
G \equiv \int_{0}^{r} s g(r) d r,
$$

where $\mathrm{g}$ is defined in Eq. (22). A noteworthy feature of Eq. (57) is its close resemblance to Eq. (45), describing a mode with $\Delta^{\prime} \mathrm{a} \sim 1$. Thus, as noted in Sec. IV C, the classical growth rate scallng, $\tau_{S}^{-3 / 5} \tau_{A}^{-2 / 5}$, is not restricted to modes with $m \geq 2$.

Another modification of the classical $m=1$ tearing mode is obtained by using the ac Ohm's law which leads to Eq. (49), but allowing $\Delta^{\prime} \lambda \sim 1$ (which can also pertain for $m>1$ ). This yields the dispersion relation 40,44 


$$
1 \alpha_{1} \omega\left(\omega-\omega_{1^{\star}}\right)\left(\omega-\omega_{e^{\star}}-\alpha_{2} \omega_{T^{*}}\right)=\gamma_{C l}{ }^{3},
$$

where $Y_{\mathrm{Cl}}$ is defined in Eq. (56). Equation (58) allows extension of the classical result to regimes in which $\omega \sim \omega_{\star} \sim \nu$. 
References

1. J.S. Dungey, Cosmic Electrodynamics (Cambridge University Press, N.Y., 1958), pp. 98-102.

2. H.P. Furth, J. Killeen and M.N. Rosenbluth, Phys. Fluids $\underline{6}, 459(1963)$.

3. H.P. Furth, Nucl. Fusion Suppl. Pt. 1, 169(1962).

4. B. Coppi, Phys. Fluids $\underline{7}, 1501(1964) ;$ Phys. Fluids $\underline{8}, 2273(1965) ;$ J. L. Johnson, J.M. Greene, and B. Coppi, Phys. Flulds $\underline{6}, 1169(1963)$.

5. G. Laval, R. Pellat and M. Vuillemin, in Plasma Physics and Controlled Nuclear Fusion Research (International Atomlc Energy Agency, Vienna, 1965), Vol. II, p. 259.

6. T.E. Stringer, Princeton Plasma Physics Laboratory Report MATT-320(1965).

7. See, for example, J.C. Hosea, C. Bobeld1jk and D.J. Grove, In Plasma Physics and Controlled Nuclear Fusion Research (International Atomic Energy Agency, Vienna, 1971), Vol. II, p. 425.

8. See, for exdiuple, S.V. Mirnov and I.B. Bemenov, in Plasma Physics and Controlled Nuclear Fusion Research (International Atomic Energy Agency, Vienna, 1971), Vol. II, p. 401; M. Murakam1, Bull. Am. Phys. Soc. 19 , $917(1974) ;$ R. Goldberg and B.S. Liley, Vacuum 18 , 6.31(1968); K. Mak1shima,

T. Tominaga, H. Tohyama, and S. Yoshikawa, Phy. Rev. Lett. 36, 142 (1976).

9. H.P. Furth, P.H. Rutherford and H. Selburg, Phys. Fluids 16, 1054 (1973).

10. J.P. Goedbloed and R.Y. Dagażan, Phys. Rev. 4, 1554 (1971).

1l. V.D. Shafranov, Zh. Tekh. Flz. 40, 24l(1970) [Sov. Phys. - Tech. Phys. $\underline{15}, 175(1970)]$.

12. See, for example, M.N. Rosenbluth, R.Y. Dagazlan, and P.H. Rutherford, Phys: Fluids 10, $1894(1973)$.

13. P.H. Rutherford and H.P. Furth, PPPL Report MATT-972 (1971).

14. R.D. Hazeltine, D. Dobrott and T.S. Wang, Phys. Flulds $\underline{18}, 1778(1975)$. 
15. J.F. Drake and Y.C. Lee, to be published in Phys. Fluids.

16. A.H. Glasser, J.M. Greene and J.L. Johnson, Phys. Fluids 18, 875(1975); Phys. Fluids $\underline{19}, 567(1976)$.

17. Liu Chen, P.H. Rutherford and W.M. Tang, Princeton Plasma Physics Laboratory Report PPPL-1338 (1977).

18. A.A: Ware, Phys. Rev. Lett. 26, 1304 (1971).

19. P.H. Rutherford, Phys. Fluids 16,1903 (1973).

20. R.B. White, D.A. Monticello, M.N. Rosenbluth and B.V. Waddell, Phys. Fluids 20, $800(1977)$.

21. B.V. Waddell, D.A. Monticello, M.N. Rosenbluth and R.B. White, Nucl. Fusion 16, $528(1976)$.

22. A. Sykes and J.A. Wesson, Phys. Rev. Lett. 37, 140(1976).

23. D. Biskamp and $\mathrm{H}$. Welter in Plasma Physlcs and Controlled Nuclear Fusion Research, Berchtesgaden, Germany (1976) (to be published).

24. B. B. Kadomtsev, Fiz. Plazmy $\underline{1}, 710(1975)$.

25. B.V. Waddell, G.L. Jahns, J.D. Callen and H.R. Hicks, Oak Ridge National Laboratory Report ORNL/TM-5840 (1977).

26. T.H. Stix, Phys, Rev. Tett. $36,521(1070) ;$ A. Rechester and T.H. S't1x, Phys. Rev. Lett. $\underline{36}, 587(1976)$.

27. T.H. Stix, Phys. Rev. Lett. $\underline{30}, 833(1973)$.

28. R.D. Hazeltine and H.R. Strauss, Phys. Rev. Lett. 37, 102 (1976).

29. M.D. Kruskal and R.M. Kulsrud, Phys. Fluids 1 , $265(1958)$.

30. G.H. Walker and J. Ford, Phys. Rev, 188, $416(1969)$.

31. H. Grad, Phys. Fluids 10, 137(1967).

32. M.N. Rosenbluth, R.Z. Sagdeev, J.B. Taylor and G.M. Zaslavski, Nucl, Fusion $\underline{6}, 297(1966)$.

33. S. von Goeler, W. Stodiek, and N. Sauthoff, Phys. Rev. Lett. 33, $1201(1974)$. 
34. See, for example, W. Stodlek, Bull. Am. Phys. Soc. 19, 930 (1974).

35. A. Rogister and G, Hasselberg, Phys. Rev. Lett. 37, 906 (1976).

36. A.A. Ware; Bull. Am. Phys. Soc. 21, 1089(1976).

37. A.A. Ware, to be published.

38. W.A. Newcomb, Ann. Phys. (N.Y.) 10, $232(1960)$.

39. B. Copp1; R. Galvao, R. Pellat, M.N. Rosenbluth and P.H. Rutherford, Princeton Plasma Physics Laboratory Report MATT-1271 (1976).

40. V. Wong, Fusion Research Center (University of Texas at Austin) Report FRC 113 (1976).

41. L. Spitzer and R. Harm, Phys. Rev. 89, 977 (1953).

42. F. L. Hinton and C.W. Horton, Phys. Fluids 14, 116(1971).

43. S.I. Braginskii, in Reviews of Plasma Physics, edited by M.A. Leontovich (Consultants Bureau, New York, 1965), Vol. I, p. 205.

44. H.R. Strauss and R.D. Hazeltine, to be published. 


\section{Figure Captions}

Figure 1. Perturbed flux surfaces are indicated by solid curves. The dashed curves are unperturbed flux surfaces. In (la), the safety factor is nonresonant and the deformation, exaggerated for clarity, is relatively

- harmless. In the resonant case of (lb), the entire shaded region is filled by a single fleld line, and the flux surfaces have been locally destroyed.

Figure 2. Magnetic field lines of the field component $\mathrm{B}_{\mathrm{n}}$, near the radius $r_{*}$, at which the sheared equilibrium field ${ }_{\sim}{ }_{0}$ is perpendicular to the plane of the figure. The field strength, $|\underset{\sim n}{B}|$, is indicated by the thickness of the lines .

Figure 3. A flux tube, distorted by a radial displacement, $\xi_{\mathrm{r}} \propto \exp (\mathbf{i} \underset{\sim}{\mathrm{k}} \cdot \underset{\sim}{\mathrm{x}})$, of the plasma "frozen" inside It. Dashed lines show the unperturbed flux tube. It is evident that the radial field perturbation would vanish if the displacement were constant along the direction of $\underset{\sim}{\mathrm{B}_{0}}$. In fact the indicated relation between $B_{r}$ and $\varepsilon_{r}$ can be inferred from the figure.

Figure 4. Perturbed version of Fig. 2. The geometry of the distortion is such that annihilation and reconnection takes place even for an infinitesimal perturbation.

Figure 5. Radial structure of the radial field perturbation $B_{r}=1 \psi$, associated with a kink-tearing mode having $\Delta^{\prime} \sim a^{-1}$. Dashed lines indicate the approximate boundaries of the tearing layer.

Figure 6. Possible forms of $\psi(r)$, near and within the tearing layer, which yleld $\Delta^{\prime} \sim \lambda^{-1}$. In case $(6 a), \psi\left(r_{s}\right)$ is quite small; in case $(6 b), \psi$ chanqes sharply within the tearing layer. 
This work was done at the suggestion of Oscar Manley, who also drew my attention to some useful references. The discussion in Section $V$, in particular, owes much to instructive conversations with H.R. Strauss and D.W. Ross. This work wa's supported by the U.S. Energy Research and Development Administration Contract EY-76-C-05-4478. 

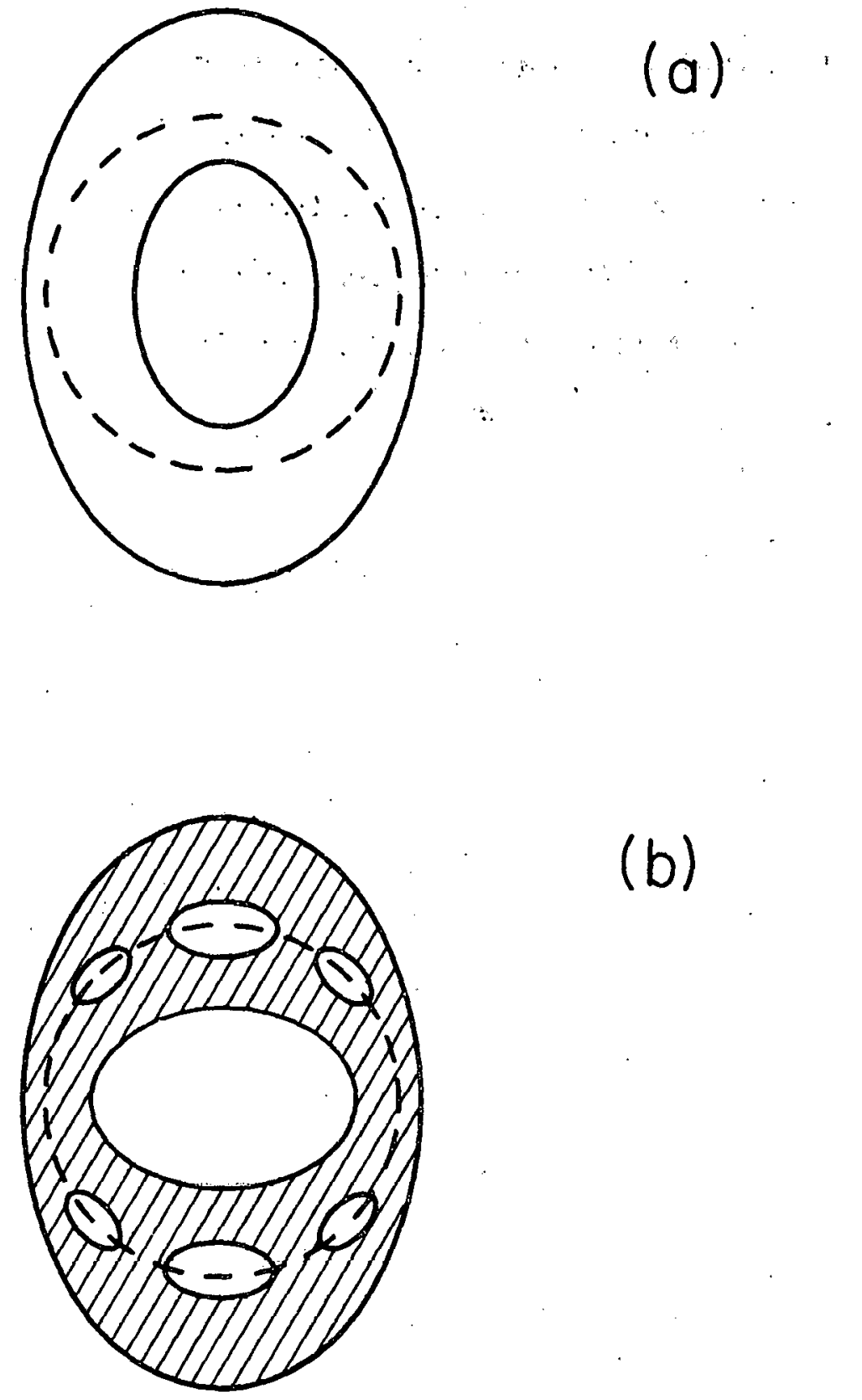

(b)

FIGURE I 


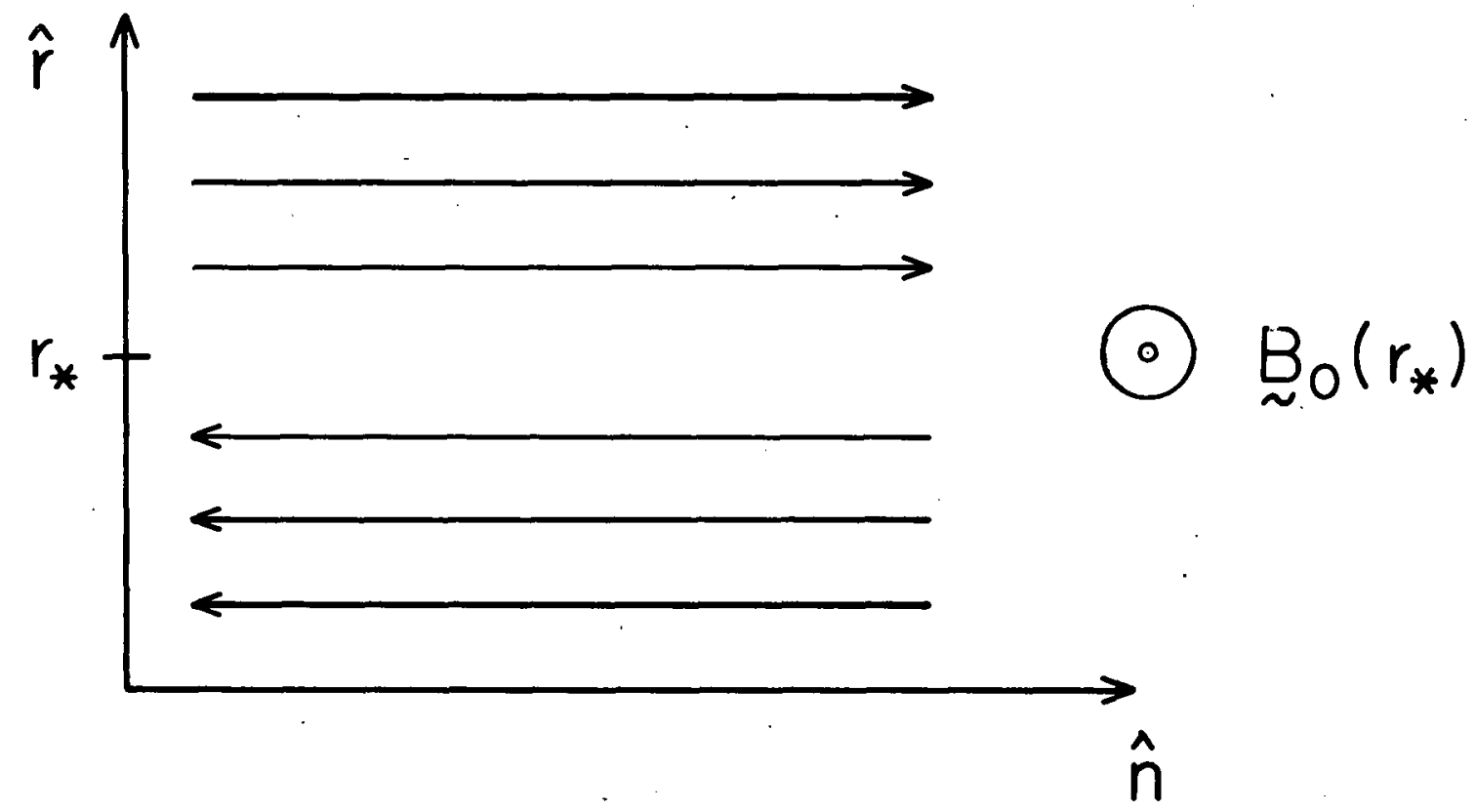

FIGURE 2 


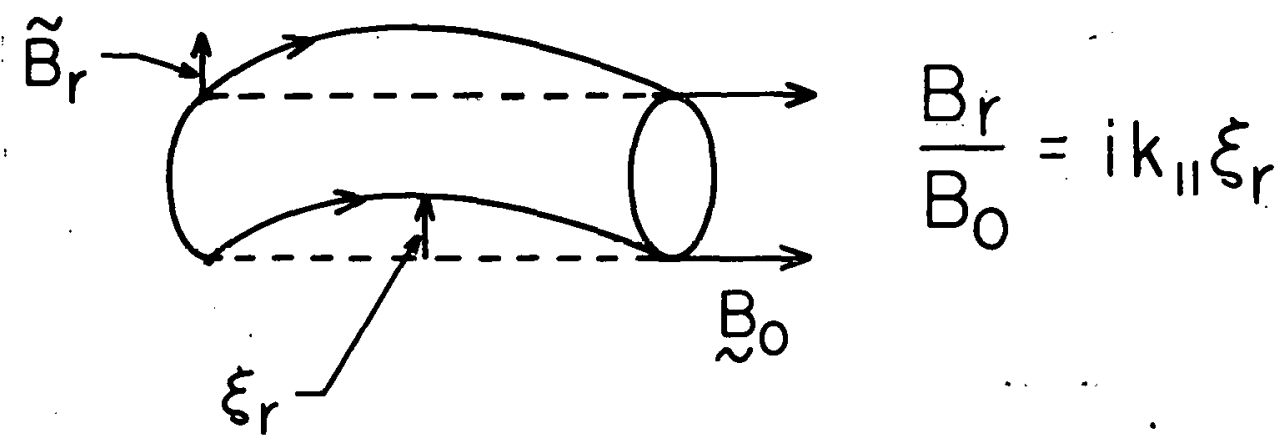

FIGURE 3 


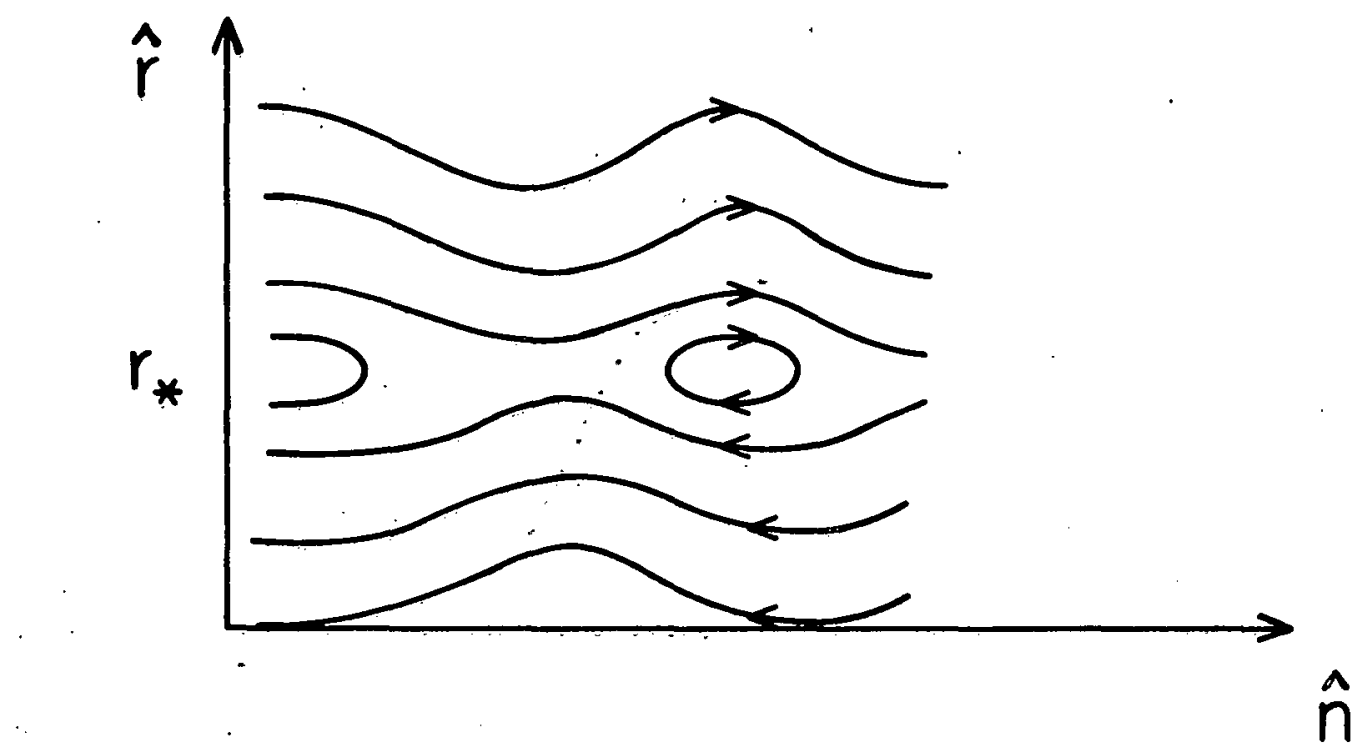

FIGURE 4 


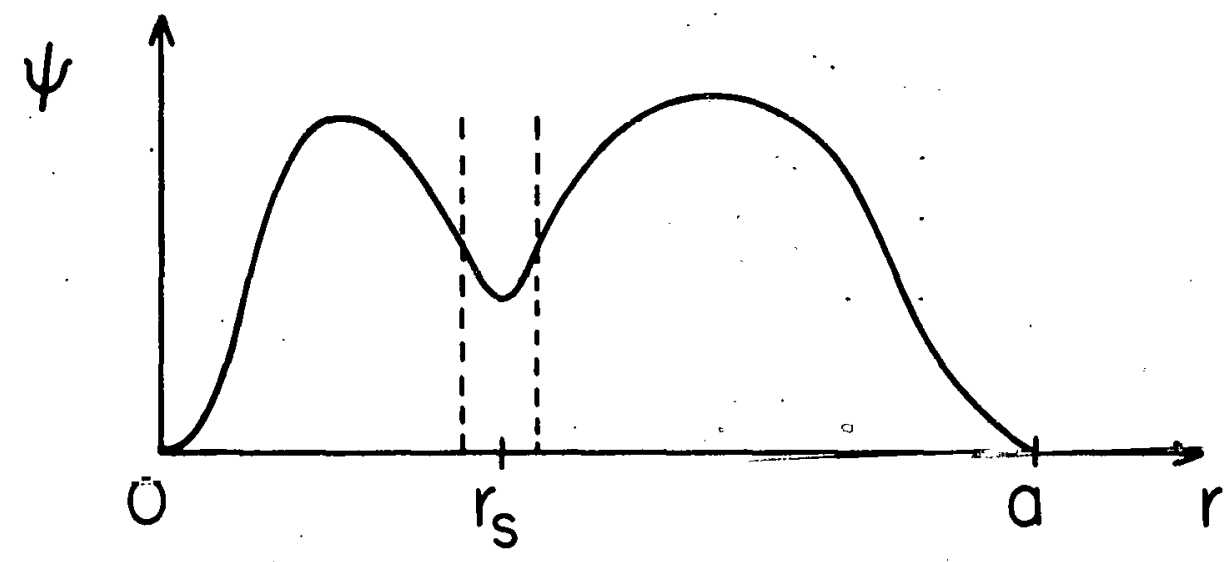

FIGURE 5 

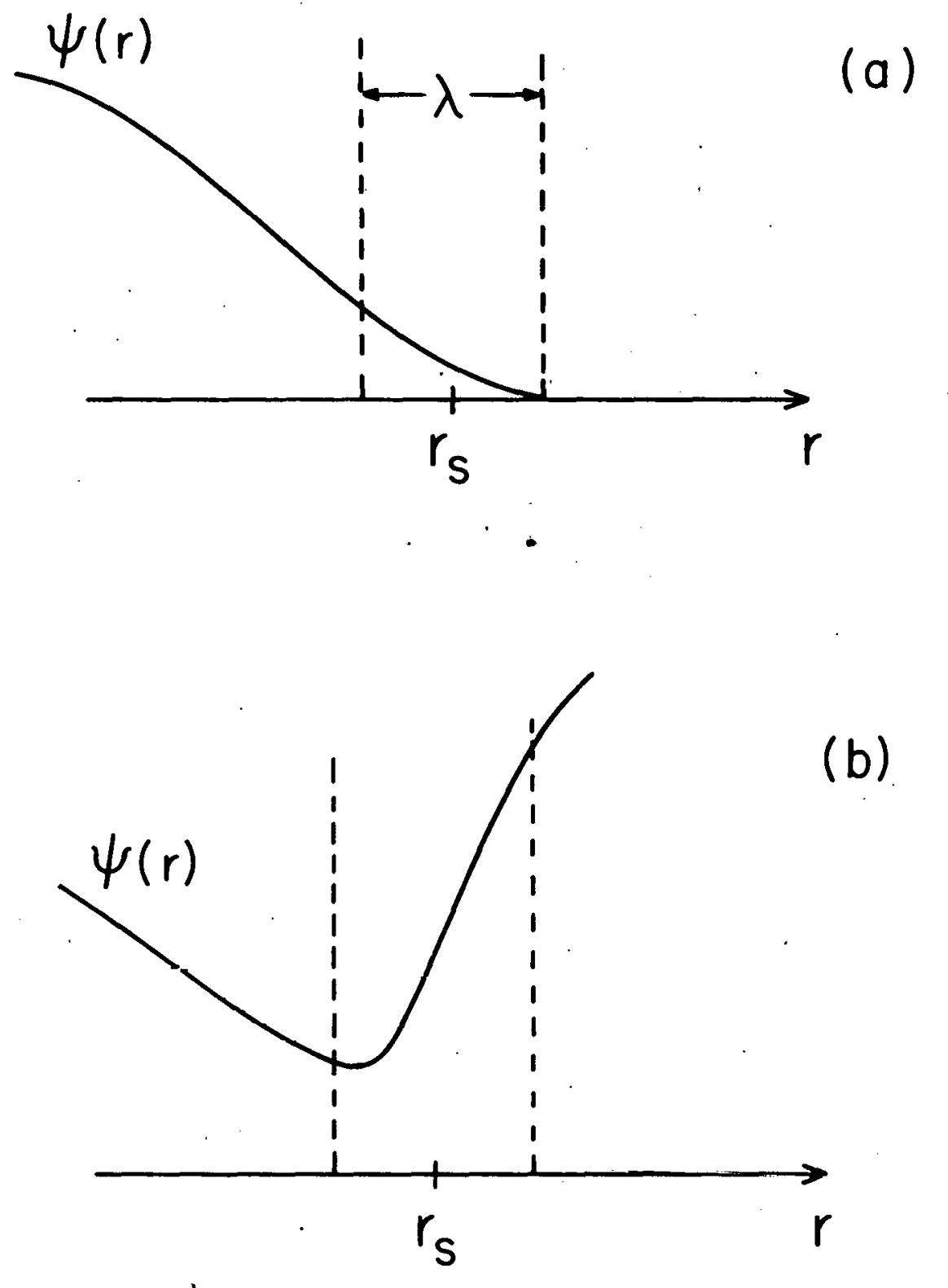

FIGURE 6 